\title{
An Enhanced Autonomous Socio-Contact Tracing System of the Spread of Contiguous Diseases
}

\author{
https://doi.org/10.3991/ijoe.v17i10.24095 \\ Nahla aljojo ${ }^{1(\varpi)}$, Alaa Musslem ${ }^{1}$, Wafa Salim ${ }^{1}$, Waad Al-Zahrani ${ }^{1}$, Sultana Al-Frran ${ }^{1}$, \\ Omar Abouola ${ }^{1}$, Areej Alshutayri ${ }^{1}$, Basma Alharbi ${ }^{1}$, Eman Aldhahri ${ }^{1}$, Azida Zainol ${ }^{1}$, \\ Hashim $\mathrm{M}$ aldwailh ${ }^{2}$ \\ ${ }^{1}$ University of Jeddah, Jeddah, Saudi Arabia \\ ${ }^{2}$ National guard Hospital, Riyadh, Saudi Arabia \\ nmaljojo@uj.edu.sa
}

\begin{abstract}
COVID-19 and other neighbouring diseases spread widely, resulting in a global epidemic that was impossible to manage and control. While numerous measures have been put in place to detect an infected person and protect uninfected areas from contracting these contagious diseases, the spread of diseases like COVID-19 continues to be rapid. As of the time of writing this paper, the number of affected people has continued to rise, and there is no clear indication of the number of people who are infected but have gone undiscovered and are spreading the infections. That is why, in order to combat the threat of contiguous disease spread, this research presented an upgraded autonomous socio-contact tracing system on a mobile platform. As a result, a generic system development process was used to create a system that allows an infected person who has been tested positive to track their electromagnetic ID card in order to determine their exact location and the risk of spreading contagious diseases. by an autonomous smart assistant that assists in describing symptoms. As a result, the system is equipped with notifications alerts for the stages of social group identification, processing, and control in order to avoid the spread of contagious diseases. The development of this system is critical for controlling epidemic diseases that are spreading over the world (particularly COVID-19) and posing a threat to people's lives. Furthermore, it contributes to a greater understanding of the seriousness of epidemic diseases and how to avoid them.
\end{abstract}

Keywords-ERP, RFID card, IoT, Arduino

\section{Introduction}

COVID illness (COVID-19) is an infectious disease caused by a newly discovered COVID. The great majority of people infected with COVID-19 will develop mild to moderate respiratory symptoms and recover without requiring special treatment [7]. Older people and people with basic medical problems including cardiovascular disease, diabetes, chronic respiratory disease, and cancer are more prone to acquire a serious illness [13]. The best way to prevent and slow transmission is to learn everything there is to know about the COVID-19 virus, the disease it produces, and how it 
spreads. Because the COVID-19 infection is conveyed primarily through droplets of saliva or release from the nose when an infected person coughs or sneezes, it's critical that you also maintain good respiratory hygiene [13]. When it comes to using technology to combat COVID-19, there are two approaches. Washing hands and social distancing are two activities recommended by proactive technology to prevent the spread of the virus. Reactive technology, which has been a key point for several countries, focuses on operations such as contact tracking and quarantine enforcement after a risk incidence has occurred [8]. COVID-19 is a global pandemic that has resulted in a large number of deaths and infected persons. To slow the spread of the COVID-19 virus, governments have imposed limitations on outdoor activities or even a population-wide quarantine. However, there is no means of knowing whether or not the sick person has committed to quarantine. As a result, epidemic diseases spread swiftly, and it became impossible to count and follow up on afflicted people.

One of the challenges we're trying to solve here is determining the location of the sick person so that he may be quarantined and the rapid spread of epidemic diseases can be slowed. As a result, we're working on a prototype system to detect the infected person's position and ensure that he's committed to quarantine. Our project's goal is to keep epidemic diseases (particularly COVID-19) from spreading over the world and endangering people's lives, as well as to promote health awareness about the importance of epidemic diseases and how to prevent them. The system will cover the following main processes: location detection to determine if the infected person has committed to quarantine, making families or individuals aware of the location of infected people around them, displaying health centres that provide epidemiological disease screening services, providing a "smart assistant" that assists the user in describing the symptoms he is experiencing, and scheduling an appointment. COVID-19 Statistics have been updated. The second approach, reactive technology, will be used to slow down the rapid spread of epidemic diseases. Throughout the literature review, the recommended solution was to build a system that can detect the location of the infected person to ensure that he committed to quarantine and slow down the rapid spread of epidemic diseases. The system includes the following features: a tracking electromagnetic ID card that allows health centres to know the exact location of an infected person if he breaks the quarantine, a smart assistant that assists the user in describing the symptoms he is experiencing and receiving a result, and an app that sends notifications to the user if he breaks the quarantine.

\section{Related work}

During the first few weeks of the coronavirus pandemic, tracking apps were hailed as a critical tool for assisting countries in their efforts to come out of lockdown. The apps would track who had been in the vicinity of an infected person using Bluetooth or GPS technology, and then notify those individuals that they had been exposed to the virus. Using the apps in conjunction with manual contact tracing and targeted quarantine, public health officials claim that they will be able to lift restrictions on the 
general population while also significantly reducing the likelihood of a second wave of infections occurring [6].

The world of information technology (IT) is improving as a result of widespread innovation, and enterprise resource planning (ERP) systems are one of them. Production management, human resources, sales, and finance are just a few of the functional modules that make up an enterprise resource planning system. ERP systems integrate all departmental functions across organisations into one single system that meets the needs of all departments in an organisation [1]. Better performance is achieved through the implementation of an ERP system, which also provides numerous benefits such as increased productivity, improved access to accurate and timely information, improved workflow, reduced reliance on paper, knowledge sharing, tight control, as well as automation of all processes by integrating and coordinating the information across all departments [1].

The advantages of enterprise resource planning systems are well known, which is why larger organisations with a large amount of data are drawn to these systems. The centralization of information through the use of a centralised database is the central theme of the ERP system [5]. Enterprise resource planning systems (ERP systems) are software modules that share a central database, and information is transferred between them once the information needs to be entered. In recent years, enterprise resource planning (ERP) systems have emerged as the most comprehensive business information systems available. ERP systems provide a solid informational foundation for operational processing as well as decision making, but they must be implemented successfully in order to be effective. A more comprehensive approach to enterprise resource planning (ERP) rather than a single piece of software, ERP brings together the functions of all departments within an organisation into one centralised system by incorporating several software applications [1]. Absher is the official individual's eServices platform in Saudi Arabia, which enables citizens and residents to access a wide range of government-sponsored services. It can be used to apply for jobs and Hajj permits, to update passport information, and to report electronic crimes. It can also be used to report fraud. Residents of Saudi Arabia can take advantage of 160 services, which include scheduling appointments, renewing passports, residents' cards, ID cards, driver's licences, and other documents, among others. It was designed and developed in 2015 with special attention paid to the security and privacy of user data and communication, and it is currently in beta testing. As a result, you can browse your profile or the profiles of your family members or labourers working for you in complete safety, as well as perform a wide range of e-Services online. Tawakkalna (Covid-19 KSA) is the official app, as shown in Figure 2.4, that has been approved by the Saudi Ministry of Health to prevent the spread of coronavirus. It was developed by the National Information Center to meet the requirements of the Saudi Ministry of Health [11].

There are numerous features included in the app, including: Provides real-time information on the number of coronavirus infections occurring throughout the Kingdom. Early detection of possible infections is made easier with this tool. In the event of an emergency during curfew, citizens and residents can request movement permits from the police department. During curfew, they should check on the status of their 
permit request. Notify them if they are within a certain distance of an infectious or isolated area. Report suspected COVID-19 cases in order to assist individuals in receiving the health care they, or others, require. Taamman is a software programme developed by the Ministry of Health in the Kingdom of Saudi Arabia that aims to strengthen the commitment of all individuals who are directed towards the confinement and follow-up of their respective cases. The app offers a variety of different services, such as the ability to schedule an appointment for the Covid-19 test [12]. There are numerous features included in the app, including: The results of the COVID-19 test. Make direct contact with 937 to request assistance. Check for symptoms on a daily basis. Update the information on who has contacted you in a positive situation. This is a collection of educational content. Isolation days are being counted down on a countdown indicator. Notifications, text messages, and automated phone calls are all used to provide alerts. Multi languages supported. A mobile application called Sehati is being developed by the Information and E-Government Authority in collaboration with the Ministry of Health and the National Health Regulatory Authority [10]. There are numerous features included in the app, including: Find a Doctor: This feature displays a list of all authorised physicians in the Kingdom of Bahrain, along with their specialties and place of employment. Medicines: Information on the medicines that are legally available in Bahrain pharmacies, as well as related information (price and supplier name). Ask a Doctor: Submit a medical question and receive a response from the appropriate medical professional. Appointments: Make an appointment at your Health Center or at the Salmaniya Medical Complex pharmacy, and check the schedules of the Salmaniya Medical Complex and the King Hamad University Hospital for availability. Licensed Pharmacies and Health Product Retailers in Bahrain: This section contains information on authorised pharmacies and health product retailers in Bahrain. Bahrain's Health-Care Facilities: This page contains information on the health-care facilities in Bahrain (hospitals, clinics, health centers). Medical Observations: Additionally, you should check the readiness status of your medical results (lab and X-ray) at Salmaniya Medical Complex and King Hamad University Hospital, as well as the result of a coronavirus (covit19) test at Salmaniya Medical Complex. Services for Birth Certificates: Request a birth certificate for each of the new born children, as well as a replacement for the existing birth certificate. Make yourself aware of the situation with the Be Aware mobile application, which aids in efforts to contain the spread of COVID-19 by speeding up contact tracing efforts. The app makes use of the location data shared by users to notify citizens and residents of a variety of events and situations [4]. There are numerous features included in the app, including: If an individual comes close to a location where an active case has been detected, or if they were in close proximity to an active case, they should be notified immediately. Track the movement of quarantine cases for a period of 14 days, and ensure that they adhere to quarantine regulations during that time. Active cases are tracked, and contact tracing updates are provided. Highlights from the COVID-19 conference. Publication of the most recent health-related recommendations issued by the Kingdom's health authorities. 


\section{$3 \quad$ Architectural Framework}

The overall architectural framework of this study is depicted in Figure 1. This research covers in great detail all of the specifics involved in the creation, interpretation, analysis, and application of architectural descriptions. An architecture framework is a set of practices that are used in a specific domain of application of computer science and is put in place to guide the process. The architecture framework for this study is a collection of practices and requirements for artefacts that describe the architecture of a system and are contained within a single presentation, which is referred to as an architecture framework. It is represented by models that depict how the microcontroller interacts with the system interface in order to fit structurally into the system and behave as if they are a component of the system. When modelling objects in a system, models are used to represent those objects. Views can be used to express a partial expression of a system from a specific perspective; however, views are not appropriate for this system, which is intended to improve the autonomous socio-contact tracing system for the spread of contiguous diseases.

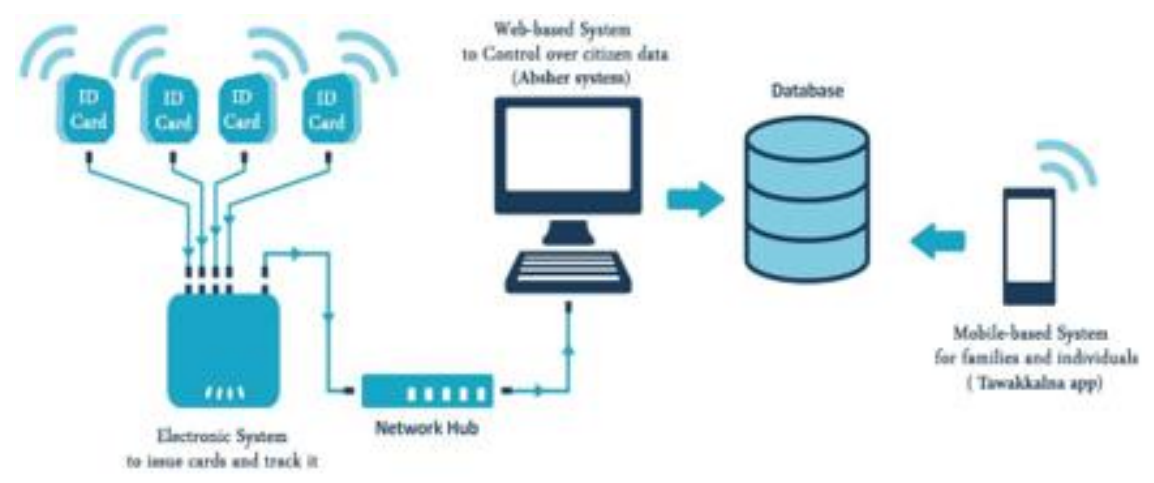

Fig. 1. The Architectural Framework

In Figure 2, you can see how Arduino, an open source programmable circuit board, can be integrated into a wide variety of makerspace projects, both simple and complex, as shown in Figure 2. This board contains a microcontroller, which can be programmed to sense and control objects in the physical world through the use of software. The Arduino is capable of interacting with a wide range of outputs, including LEDs, motors, and displays, by responding to sensors and inputs received from the board. Arduino has become a very popular choice for makers and makerspaces who want to create interactive hardware projects because of its flexibility and low cost [3]. (See Fig. 3 for an example.) Arduino distinguishes itself from other platforms available on the market by offering the following features: It is a multiplatform environment that can be used on multiple platforms, including Windows, Macintosh, and Linux. It is built on the Processing programming integrated development environment (IDE), which is a simple-to-use development environment popular among artists and designers. Instead of using a serial port, you programme it using a USB cable. Because 
many modern computers do not have serial ports, this feature is extremely beneficial. Arduino is open source hardware and software, which means that if you want, you can download the circuit diagram, purchase all of the components, and assemble your own without having to pay anything to the Arduino developers. The hardware is reasonably priced. The USB board is approximately $\$ 35$ in price. As a result, you have the resources to complete several projects. There is a vibrant user community, so you will find plenty of people who are willing to assist you. The Arduino Project was developed in an educational environment, making it a great choice for newcomers who want to get things up and running as quickly as possible.

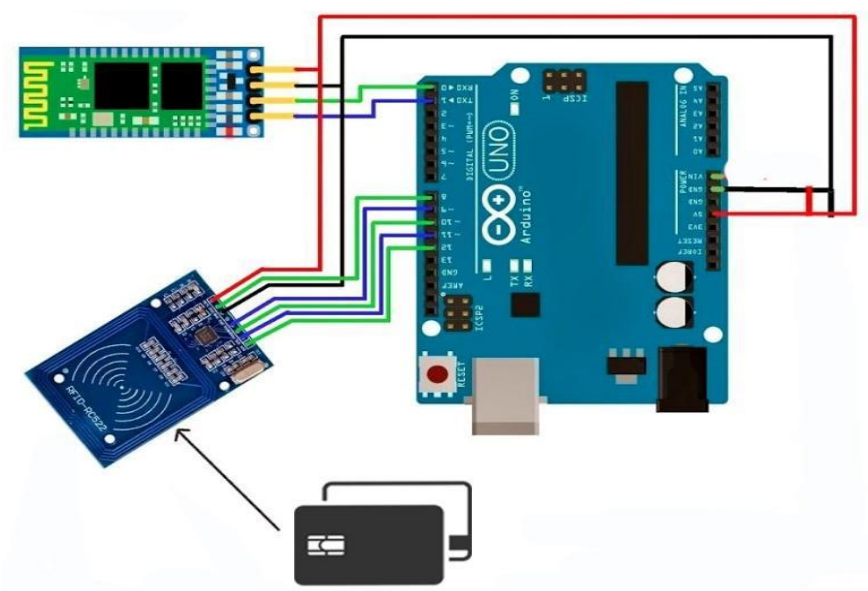

Fig. 2. IoT Based RFID tracking system Using Arduino Diagram

\section{Materials and Methods}

Four stages have been completed in order to learn how to solve the problem of detecting the location of the infected person in order to ensure that he is committed to quarantine and to slow the rapid spread of epidemic disease.

First and foremost, we distributed a questionnaire prior to developing the system in order to gather information about some critical issues, such as the community's acceptance of the concept in question. All ages and both genders participated in the survey in Jeddah, Saudi Arabia, which was conducted in Arabic. A Google forms survey was used, and we received 225 responses. There were 20 questions total, and we used Google forms to collect the information. A significant number of respondents (68.8 percent) expressed support for the idea of adding a new feature to the Absher system that would allow the health supervisor to detect whether or not the infected person had committed to quarantine after being exposed to the infection. The idea of updating electronic ID cards to allow the government to track infected people with corona is supported by approximately 87.5 percent of respondents. In our research, we discovered that the community accepts the concept of a system that can detect the 
location of an infected person in order to ensure that he has committed to quarantine, and that the community believes it is a good idea that would be beneficial to them.

\subsection{Design and Development}

For this project, we created a paper-based prototype to examine web content and interaction techniques from the perspectives of designers and users alike. Second, gather feedback from users on the design process so that it can be taken into consideration during the actual implementation of the system. Third, by collecting data on user mistakes and comments, usability problems can be identified at an early stage of design, allowing for significant savings in resources that would otherwise be spent on flawed products. Storyboarding is a technique used to create a paper-based prototype of a system that is built using one of the techniques used to display the screens. This is illustrated in Figure 3, which depicts the system's low reliability.

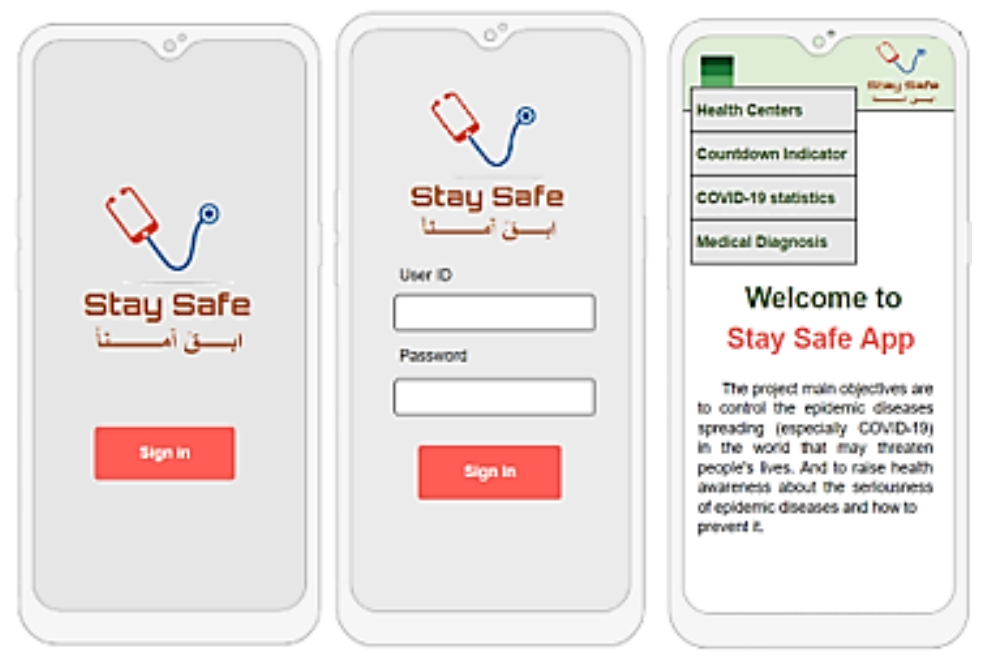

Fig. 3. Sign In in app system, index in app system in the main menus in app system

Arduino tools and the Arduino Software IDE were used to construct the RFID card model. Code for the Arduino Software Integrated Development Environment (IDE) is shown in the following example. Ultrasonic sensors (as shown in figure 4) and an Arduino Uno microcontroller were used in the hardware implementation. Additional reasons for deciding on the Arduino include the following: It is relatively cheap to purchase an Arduino board. Operating systems such as Macintosh, Linux, and Windows can all be used to run the Arduino Software Development Environment (IDE). It is possible to programed in the C++ language [2]. To detect people in the vicinity of a card reader, tow sensors have been installed next to the reader's location. In addition to having 14 digital input/output pins, Arduino Uno is a microcontroller board that can be programmed using the Arduino Software IDE [2]. Figures 4 and 5 illustrate the connection between an RFID card and a card reader. 


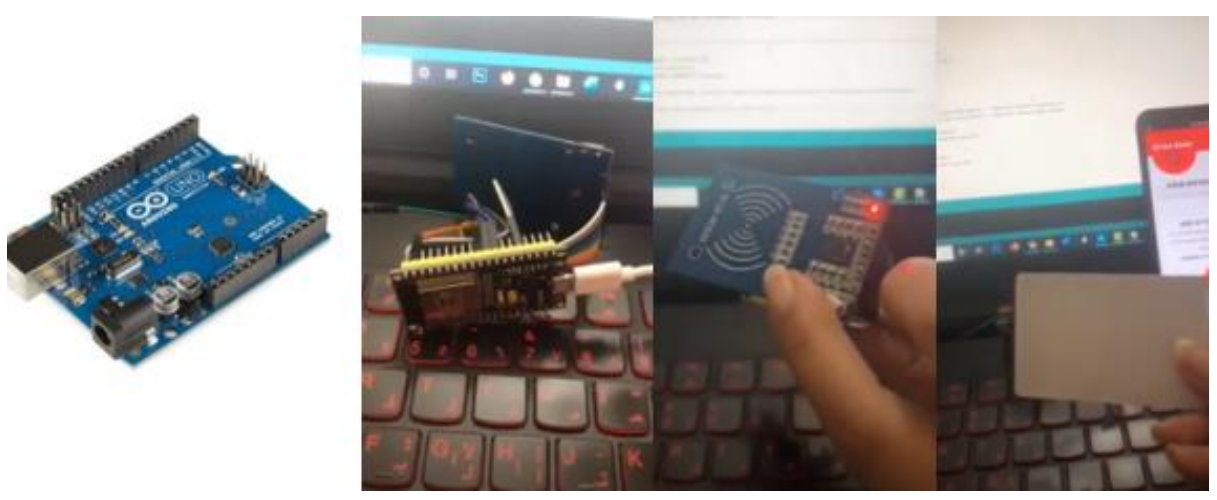

Fig. 4. Arduino Uno Microcontroller, Card reader and RFID card

\subsection{Results and Discussion}

The implementation process is based on the review and analysis of some systems and applications, and it is dependent on how the system will provide useful information that will support the users and simplify their requirements. The Website, the health centre, the Ministry of the Interior, and the application for individuals are all displayed during the implementation process.

When a user visits a website for the first time, the home page is displayed. For the user to be able to access system information, this page contains a login form, which must be completed before accessing the system information. Following that is the Health Center and the Welcome Page. When an employee of the health centre logs into the system, this page will be displayed to them. There are four main buttons on this page. The first button allows you to enter test results, and the second button allows you to detect the location of the test results. The third button allows you to see all of your scheduled appointments. The fourth button allows you to see the results of the test. This is followed by the profile page, which displays the user's personal information and allows him or her to make changes to that information. This is followed by an appointment to view the profile page. This page contains information about It will be possible for the health centre employee to view the appointments that have been reserved for the users through this page. Add test result is the feature that comes after it. User test results will be added to this page by a health centre employee working on this page for them. Viewing the test results is the next step. By entering the user name or the test name on this page, the health centre employee will be able to view the results of the test. The Ministry of the Interior is the second on the list.

The Ministry of the Interior welcome page appears after an employee of the Ministry of the Interior logs into the system for the first time. There are three main buttons on this page. The first button is used to update ID cards, and the second button is used to determine the location of the device. The third button allows you to see the results of the test. This is followed by the "Update ID cards" option page, from which an employee of the Ministry of the Interior can make changes to the user's identification cards and other documents. The next page is followed by the view test result link, 
which allows employees of the Ministry of the Interior to view the test results by entering their user name or the test name into the appropriate field. Next, the Application for Individuals home interface is displayed to the user, where he has the option to open the application start page, which will be displayed to him with the sign-in button that appears in the centre. The next page is the Sign in page, which appears when the user clicks on the sign-in button and a sign-in page is displayed. Absher users will be able to sign in immediately by entering their id and password because they will be using the same account that they already have in Absher. This is followed by a welcome interface that displays the main menu, which contains a number of different options (home, profile, health centers, countdown indicator, COVID-19 statistics, medical diagnosis, notification) the application's scope of operation.

\subsection{Experimental Evaluation}

The experimental evaluation of this study is centred on the application of the system in question. Hardware and software, including a laptop or desktop computer, as well as a smartphone, were required in order to run the entire system. Arduino Uno, RFID reader, and electromagnet card are all examples of open source hardware. The software operating system is comprised of the following components: Microsoft Windows (Windows 7, Windows 8, and Windows 10) Android. To prepare the document, the following software was used: Microsoft Office Word and Microsoft Office Excel. Later, functional testing was used for evaluating and verifying the system's functions in order to determine whether or not the system conforms to the specified requirements; the testing procedures used included unit and integration testing as well as compatibility testing as well as usability testing. Known as unit testing, it is a software development process in which the smallest testable parts of an application (referred to as units) are individually and independently scrutinised to ensure that they function properly. This testing is carried out in order to determine whether or not all of the functions are functioning as expected. The test process was carried out on the parents in accordance with the Functional Requirements described in the Analysis chapter. It is a type of software testing in which individual units are combined and tested as a group. It is a subset of unit testing. The goal of this level of testing is to identify and isolate faults that occur during the interaction of integrated units. Integration Testing is made possible through the use of test scenarios and test results. To determine whether our system is compatible with two different computing environments, we will run it through its paces on two different devices and with two different versions of the same software. By testing the product from the user's perspective, usability testing determines whether the product is useful to use or not. It also ensures that the user understands everything in the system and measures how easy it is to use. We evaluated the usability of an Android application designed to track the spread of epidemiological diseases through the use of Arduino RFID card functions, which we developed. McLaughin and Skinner's principles for determining the usability of application criteria, which are comprised of six criteria, are described by Whitehead (2006). 


\section{$5 \quad$ Conclusion and Future Work}

The android application to track the spread of epidemiological diseases using an Arduino RFID card was developed to assist health supervisors in tracking infected cases and containing the spread of disease, as well as to educate the general public about the presence of infected people in their immediate vicinity, among other things. To summarise our experience in developing this application, we have done the following. In this project, we attempted to explain the concept and formulate it in the most effective way possible, after which we looked more closely at competitors and similar actions. Then we got to work looking at our users' opinions and analysing the data we had collected from them in order to better understand and meet their needs. It was then that we began to work on the actual implementation of the system, which we tried and tested to ensure that it was of high quality. We have encountered a number of difficulties throughout this project. We found it difficult to learn how to create an app on our own and went on a search for a tutorial to help us improve our selflearning skills. In addition, we encountered a few coding issues. In addition, when we were developing our application pages, we encountered the challenge of figuring out how to make an application that would work on a mobile device as well as an application that would support REID card technology. Because of the large number of functions that were included in our project, it has become increasingly complex over time, and the limited time available to us has not been helpful. We were required to complete as many project functions as we possibly could, but in the end, we completed the most important functions and left the less important functions and details to be completed in the future. There are numerous areas that can be improved, as well as additional features that can be added. For example, the system could be implemented as a smartphone application for use with other phone platforms. A web-based version of the system could be made available. Include some extra features, such as the ability to call a doctor. Increase the number of diseases in the application. The application administrator could be added to the system as a future enhancement. Alternatively, the system could be uploaded to Google Play and integrated with the Absher platform. Extend the test phase to additional cities.

\section{Acknowledgment}

First of all, thank to Allah the most merciful for endowing us with health, patience, knowledge, and strength to complete this project, then we would like to express our sincere gratitude to our supervisor Dr. Nahla Aljojo for the continuous support.

\section{$7 \quad$ References}

[1] Abrar Ullah, Khalil MD Nor, Rohaizat Baharun, Muhammad Yasir, 2018. Overview of Enterprise Resource Planning (ERP) System in Higher Education Institutions (HEIs). Journal of Computational and Theoretical Nanoscience, 24(6), pp. 4399-4406. https:// doi.org/10.1166/asl.2018.11614 
[2] Arduino, 2018. Arduino Software (IDE). [Online] Available at: https://www.arduino. cc/en/Guide/Environment [Accessed 710 2020].

[3] Banzi, M., 2011. Getting Started With Arduino. Second Edition ed. s.1.:O'Reilly Media, Inc.

[4] BeAware, 2020. BeAware Bahrain - Information \& eGovernment Authority. [Online] Available at: https://play.google.com/store/apps/details?id=bh.bahrain.corona.tracker [Accessed 229 2020].

[5] Benders, J., Batenburg, R., \& Van der Blonk, H. (2006). Sticking to standards; technical and other isomorphic pressures in deploying ERP-systems. Information \& Management, 43(2), 194-203. https://doi.org/10.1016/j.im.2005.06.002

[6] Gold, H., 2020. Tracking apps were supposed to help beat the pandemic. What happened to them?. [Online] Available at: https://edition.cnn.com/2020/06/05/tech/coronavirustracking-apps/index.html [Accessed 219 2020].

[7] Ortiz-Prado, E., Simbaña-Rivera, K., Gómez-Barreno, L., Rubio-Neira, M., Guaman, L. P., Kyriakidis, N. C., ... \& López-Cortés, A. (2020). Clinical, molecular, and epidemiological characterization of the SARS-CoV-2 virus and the Coronavirus Disease 2019 (COVID-19), a comprehensive literature review. Diagnostic microbiology and infectious disease, 98(1), 115094. https://doi.org/10.1016/j.diagmicrobio.2020.115094

[8] Panetta, K., 2020. Reduce the spread of COVID-19 using gamification and technology.. [Online] Available at: https://www.gartner.com/smarterwithgartner/how-technology-cancurb-the-spread-of-covid 19/\#: :text=Proactive\%20technology\%20encourages $\% 20$ behaviors\%20to,contact\%20tracing\%20and\%20quarantine\%20enforcement. [Accessed 99 2020].

[9] Questionpro, 2017. Data Collection: Definition, Methods, Example and Design. [Online] Available at: https://www.questionpro.com/blog/data-collection/ [Accessed 10 2020].

[10] Sehati, 2020. Sehati - Information \& eGovernment Authority. [Online] Available at: https://play.google.com/store/apps/details?id=bh.bahrain.Sehati [Accessed 229 2020].

[11] Tawakkalna, 2020. Tawakkalna (Covid-19 KSA) - National Information Center. [Online] Available at: https://play.google.com/store/apps/details?id=sa.gov.nic.tawakkalna [Accessed 229 2020].

[12] Tetamman, 2020. Ministry of Health - ( Tetamman ) تطمن. [Online] Available at: https://play.google.com/store/apps/details?id=com.tetaman.home [Accessed 229 2020].

[13] WHO.int, 2020. Coronavirus. [Online] Available at: https://www.who.int/healthtopics/coronavirus\#tab=tab_1 [Accessed 99 2020].

[14] World Health Organization. (2020). Addressing noncommunicable diseases in the COVID-19 response.

\section{Authors}

Nahla Aljojo obtained her PhD in Computing at Portsmouth University. She was Associate Professor at Faculty of Computing and Information Technology Information System Department - King AbdulAziz University, Jeddah, Saudi Arabia. She is working currently as Associate Professor at College of Computer science and Engineering, Information system and Information Technology Department, University of Jeddah, Jeddah, Saudi Arabia. Her research interests include adaptivity in web-based Educational Systems, E-business, leadership's studies, information security and Data Integrity, E-Learning, Education, Machine Learning, AI, and Deep Learning. 
Alaa Musslem in 2020 with a Bachelor of Information systems and Technology, from the College of Computer Science and Engineering at the University of Jeddah, Jeddah, Saudi Arabia (Email: amusslem.stu@uj.edu.sa).

Wafa Salim in 2020 with a Bachelor of Information systems and Technology, from the College of Computer Science and Engineering at the University of Jeddah, Jeddah, Saudi Arabia (Email: wsalim.stu@uj.edu.sa).

Waad Al-Zahrani in 2020 with a Bachelor of Information systems and Technology, from the College of Computer Science and Engineering at the University of Jeddah, Jeddah, Saudi Arabia (Email: walzahrani0182.stu@uj.edu.sa).

Sultana Al-Frranin in 2020 with a Bachelor of Information systems and Technology, from the College of Computer Science and Engineering at the University of Jeddah, Jeddah, Saudi Arabia (Email: sf0001.stu@uj.edu.sa).

Omar Abouola is an Assistance professor of College of Computer science and Engineering, Information system and Information Technology Department, University of Jeddah, Jeddah, Saudi Arabia.

Areej Alshutayri is an Assistant Professor in the department of computer science and Artificial Intelligence at the university of Jeddah. Areej collected and created a social media Arabic dialect text corpus (SMADC) using Twitter, Facebook, and Online newspapers. Areej's research interests in using Artificial Intelligence which includes machine learning and natural language processing to understand languages especially Arabic language and its dialects.

Basma Alharbi received her Ph.D. degree in Computer Science from King Abdullah University of Science and Technology (KAUST), Thuwal, Saudi Arabia in 2017. She received her M.Sc. degree in Computer Science from Durham University, Durham, United Kingdom in 2009, and her B.Sc.degree in Computer Science from Effat University, Jeddah, Saudi Arabia in 2008. Dr. Alharbi is currently an assistant professor at the Computer Science and AI department, in the College of Computer Science and Engineering, at the University of Jeddah, Jeddah, Saudi Arabia.

Eman Aldhahri received her Ph.D. degree in Computer Science from University of Memphis, Memphis, United States of America in 2019. She received her M.Sc. degree in Computer Science from Southern Illinois University, Carbondale, United States of America in 2014, and her B.Sc. Degree in Computer Science from Taibah University, Al-madinah, Saudi Arabia in 2006. Dr. Aldhahri is currently an assistant professor at the Computer Science and AI department, in the College of Computer Science and Engineering, at the University of Jeddah, Jeddah, Saudi Arabia.

Azida Zainol received a PhD in Computer Science from Bangor University, United Kingdom. Since then, she is actively involved in research development. Her main areas of research interest are secure software development and software engineering. Currently, she is an Assistant Professor at College of Computer Science and Engineering, University of Jeddah, Saudi Arabia.

Hashim M Aldwailh is Saudi board certified in anesthesia, Bachelor of Medicine, Bachelor of Surgery (MBBS). Obstetric Anesthsia Fellow in National Guard hospital in Riyadh, Saudia Arabia.

Article submitted 2021-05-18. Resubmitted 2021-08-05. Final acceptance 2021-08-15. Final version published as submitted by the authors. 